Check for updates

Cite this: RSC Adv., 2018, 8, 31911

\title{
Synergistic delivery of bFGF and BMP-2 from poly(L-lactic-co-glycolic acid)/graphene oxide/ hydroxyapatite nanofibre scaffolds for bone tissue engineering applications
}

\author{
Xiansheng Ren, ${ }^{\text {ab }}$ Qinyi Liu, ${ }^{\text {b }}$ Shuang Zheng, ${ }^{\text {b }}$ Jiaqi Zhu, ${ }^{\mathrm{c}}$ Zhiping Qi, ${ }^{\mathrm{b}}$ Chuan Fu, \\ Xiaoyu Yang ${ }^{\star b}$ and Yan Zhao (D) $\dagger^{\star a}$
}

One of the goals of bone tissue engineering is to create scaffolds with excellent biocompatibility, osteoinductive ability and mechanical properties. The application of bioactive proteins, such as bone morphogenetic protein (BMP)-2 and basic fibroblast growth factor (bFGF), has been showed to be an effective way to improve the osteoinductivity and biocompatibility of bone scaffold materials. Therefore, the development of novel materials capable of delivering multiple growth factors is urgent and essential for bone defect repair. In this study, a composite nanofibre scaffold composed of poly(L-lactic-COglycolic acid) (PLGA), hydroxyapatite (HA), and graphene oxide (GO) has been fabricated to deliver basic fibroblast growth factor (bFGF) and bone morphogenetic protein-2 (BMP-2) simultaneously. The data show that the incorporation of GO and HA into PLGA nanofibres significantly improved the mechanical properties and hydrophilicity of the nanofibre scaffolds. More importantly, compared to PLGA and PLGA/ HA nanofibre scaffolds, the PLGA/HA/GO nanofibre scaffolds could more efficiently immobilize bFGF and BMP-2. Moreover, biological assays indicated that the loaded bFGF and BMP-2 loaded in the composite nanofibre scaffolds have a synergistic differentiation effect on the cell adhesion, proliferation, and osteogenesis differentiation of MC3T3-E1 cells. In contrast to the PLGA/HA/GO/bFGF and PLGA/HA/ GO/BMP-2 nanofibre scaffolds, the PLGA/HA/GO/bFGF/BMP-2 scaffolds have shown higher ALP activity and higher expression levels of osteogenesis-related genes. In summary, our findings indicated that the incorporation of GO into nanofibre scaffolds is an effective method to immobilize growth factors onto biomaterial surfaces, and the synergistic effects of a combination of BMP-2 and bFGF may have potential use in bone regenerative therapeutics.

Received 19th June 2018

Accepted 26th August 2018

DOI: $10.1039 / c 8 \mathrm{ra05250f}$

rsc.li/rsc-advances

\section{Introduction}

Bone tissue engineering shows great potential in developing novel biomaterials as bone substitutes and grafts for bone regeneration therapies. An ideal bone tissue engineering scaffold should have excellent biocompatibility, biological activity and mechanical properties that can mimic the extracellular matrix (ECM) and regulate cell function. ${ }^{1}$ Growth factors refer to naturally occurring proteins capable of targeting specific cellular receptors and triggering various cellular processes. Currently, numerous growth factors are known to induce

${ }^{a}$ Physical Examination Center, The Second Hospital of Jilin University, Ziqiang Street No. 218, Changchun TX: 130041, PR China.E-mail: zhaoyano1@jlu.edu.cn ${ }^{b}$ Department of Orthopedic Surgery, The Second Hospital of Jilin University, Ziqiang Street No. 218, Changchun TX: 130041, PR China.E-mail: yangxiaoy@jlu.edu.cn ${ }^{c}$ Department of Gynecology and Obstetrics, The First Hospital of Jilin University, Xinmin Street No. 71, Changchun TX: 130000, PR China

$\dagger$ Current address: Physical Examination Center, The Second Hospital of Jilin University, Ziqiang Street No. 218, Changchun TX: 130041, PR China. osteogenic differentiation and are used in the treatment of bone defects. ${ }^{2-4}$ However, many kinds of growth factor proteins are easily degraded by proteinases in the human body, and an overabundance of growth factors might lead to undesirable adverse effects, such as ectopic bone formation, osteolysis, immune responses, and tumourigenesis. ${ }^{5,6}$ One strategy to solve this problem is combining bone scaffold materials with growth factors, which can not only reduce the abundance of growth factors but also improve the bioactivity of bone scaffold materials. At present, many studies have reported that incorporating growth factors into bone scaffold materials can significantly induce cellular osteogenic differentiation as well as enhancing bone formation. ${ }^{2,7,8}$

Among the growth factors, BMP-2 is one of the most important members of the osteogenesis related growth factors and is widely considered to be the strongest factor for osteogenic induction. Multiple studies have demonstrated that BMP2 could effectively regulate the adhesion and osteogenic differentiation of cells in vitro and induce ectopic osteogenesis in 
vivo. ${ }^{9-11}$ Furthermore, BMP-2 was approved by the United States Food and Drug Administration for clinical applications. ${ }^{12}$ A bone scaffold materials with a BMP-2 delivery system was found to be effective in the repair of critical sized segmental bone defects and to improve the osteogeneic activity of bone scaffold materials. The process of bone formation starts with the induction of BMP-2, which is subject to comprehensive regulation by several growth factors. Therefore, enhanced bone regeneration can be achieved by combined treatment with BMP2 and other growth factors compared to that achieved by the use of BMP-2 alone. As a member of the fibroblast growth factor family, bFGF is an important regulator of the proliferation, differentiation and migration of various types of cells such as vascular cells and osteoblasts. ${ }^{\mathbf{1 3 , 1 4}}$ Therefore, bFGF has therefore been recognized as an important growth factor in the process of bone fracture healing. Previous studies demonstrated that the bFGF can promote bone regeneration and osseointegration during fracture healing. ${ }^{15,16}$ The present study have demonstrated that the delivery of both BMP-2 and bFGF together could enhance new bone formation and vascularization compared with that achieved by the delivery of BMP-2 or bFGF alone. ${ }^{14,15,17}$ For the above reasons, the use of bFGF and BMP-2 in combination to improve the osteointegration of bone scaffold materials has garnered our attention.

Currently, there are many methods of combining growth factors into polymer bone scaffolds, including the direct blending method and the immobilization method. ${ }^{18-21}$ However, the direct blending method will damage the bioactivity of growth factors because of the used of organic solvents. Furthermore, the poor hydrophilicity and the lack of functional groups on the polymers in the bone scaffold often result in a decreased growth factor loading efficiency; thus, the growth factors could not be tightly bound to the polymer bone scaffolds by the surface immobilization method. Because of the above reasons, many methods for the uniformly immobilization of growth factors have been evaluated, such as plasma treatment, photoreactive gelatine and poly(dopamine) coating. ${ }^{7,22,23}$ Graphene, an atomically thick sheet with $\mathrm{sp}^{2}$ carbon atoms in a honeycomb structure, possesses unique physicochemical characteristics such as extraordinary mechanical properties and hydrophilic functional groups and a high specific surface area. Because of the excellent properties, graphene and its derivatives have been intensively studied for biomedical applications, such as drug delivery carriers, imaging agents, cancer photothermal therapy agents and tissue engineering scaffolds components. ${ }^{24-27}$ Previous studies had indicated that the mechanical properties and biocompatibility of polymers bone scaffolds doped with GO were significantly better than those of pure scaffolds. ${ }^{28,29}$ Additionally, GO is composed of hydrophobic $\pi$ domains in the core region and ionized groups along the edges. These features enhance its binding affinity to growth factors via hydrophobic and electrostatic interactions. All these positive attributes contribute to making GO more efficient as a drug carrier than are other carbonaceous nanomaterials. Numerous studies have found that GO can serve as an intermediate for the conjugation of growth factors onto polymer scaffold surfaces. ${ }^{30}$ Furthermore, previous studies showed that the incorporation of
GO into polymer scaffolds can significantly improve the proteinand inducer-adsorption ability, thus leading to the enhancement of both cell proliferation and osteogenic differentiation. ${ }^{31,32}$ Meanwhile, GO was able to facilitate the binding of calcium and promote the mineralization process, which can regulate cell osteogenic differentiation. ${ }^{33}$ According to the above reasons, we believe that the incorporation of GO can improve the biological properties of polymer scaffolds and effectively attach the growth factors to the polymer scaffold surface.

In this manuscript, the electrospun nanofibrous scaffolds that were was formed with PLGA, HA and GO were used as the growth matrix for MC3T3-E1 cells and as the sustained-release carrier for the growth factors, BMP-2 and bFGF, which were expected to generate more effective osteogenesis. The aim of this study was to improve the cell affinity and osteoinductive ability of nanofibre scaffold surfaces with the immobilization of BMP-2 and bFGF via the GO. Alkaline phosphatase (ALP) activity, mineral deposition, and bone-related protein expression assays were performed to explore the osteoinductive abilities of different nanofibre scaffolds. Our findings demonstrated that the new BMP-2 and bFGF immobilized PLGA/HA/GO nanofibre scaffold was an excellent biomaterial for inducing bone regeneration.

\section{Experimental}

\section{Materials}

Poly(L-lactic-co-glycolic acid), with an LA/GA ratio of $75: 25$ and a molecular weight of $150000 \mathrm{~g} \mathrm{~mol}^{-1}$, was purchased from SinoBiomaterials Co.,Ltd. GO was purchased from Chengdu Organic Chemicals Co. Ltd, China (thickness: 0.55-1.2 nm, diameter: $0.5-3 \mu \mathrm{m}$ ) and HA powder was obtained from the Changchun Institute of Applied Chemistry, Chinese Academy of Sciences (CIAC, China). bFGF was purchased from Peprotech, USA. BMP-2 was purchased from UB Biotech. HA powder was obtained from Nanjing Emperor Nano Material Co., Ltd. 1,1,1,3,3,3-hexafluoroisopropanol (HFIP) and 3-(4,5-dimethyl-2thiazolyl)-2,5-diphenyl-2- $H$-tetrazolium bromide (MTT) were purchased from Sigma-Aldrich (USA).

\section{Fabrication of PLGA/HA/GO nanofibre scaffolds}

PLGA/HA/GO nanofibre scaffolds were fabricated by electrospinning. Briefly, PLGA (20 wt $\%$ ) and HA were dissolved in 1,1,1,3,3,3-hexafluoro-2-propanol (HFIP) to prepare the composite solution of PLGA/HA (PLGA : HA = $9: 1$ ). Then, GO was added to the above solutions to obtain a $2 \mathrm{wt} \%$ blend, and the mixture was stirred for $24 \mathrm{~h}$ at room temperature. Finally, the mixture solutions of PLGA, HA and GO was used for electrospinning. The nanofibre scaffolds were fabricated by electrospinning at an applied voltage of $20 \mathrm{kV}$ with a dispensing rate of $1 \mathrm{~mL} \mathrm{~h}^{-1}$, an air gap distance of $20 \mathrm{~cm}$, and an injection needle diameter of $0.61 \mathrm{~mm}$. Finally, the PLGA/HA porous scaffolds were obtained after being vacuum dried to remove the residual solvent. Pure PLGA and PLGA/HA were also prepared under the same conditions. 


\section{Characterization of the nanofibre scaffolds}

The different nanofibre scaffolds were coated with gold, and observed under a scanning electron microscope (SEM, XL 30 ESEM-FEG, FEI). The average diameter of the fibres was determined from that of 100 fibers by imaging and analysis with Image J software. The static water contact angle was used to evaluate the surface wettability of the nanofibre scaffolds using a Kruss GmbH DSA $100 \mathrm{Mk} 2$ goniometer. X-ray diffraction (XRD, D8 ADVANCE, Germany) and energy-dispersive X-ray spectroscopy (EDX, Philips, XL-30W/TMP, Japan) were used to detect GO and HA in the nanofibre scaffolds. To investigate the mechanical properties, strips of different nanofibre scaffolds of width $\times$ initial length $=10 \times 30 \mathrm{~mm}$ were tested with a universal mechanical testing machine (Instron 1121, UK).

\section{Surface immobilization of bFGF and BMP-2}

The immobilization of bFGF and BMP-2 on the surface of the nanofibre scaffolds was accomplished by physical binding between GO and the growth factors. Briefly, to immobilize the growth factors, the PLGA/HA and PLGA/GO/HA nanofibre scaffolds were immersed in a PBS solution containing bFGF (100 ng $\left.\mathrm{mL}^{-1}\right)$ and BMP-2 (200 ng mL $\mathrm{mL}^{-1}$ ) to immobilize growth factors for $90 \mathrm{~min}$ at $37^{\circ} \mathrm{C}$, respectively. After two washes with deionized water to remove any unbound growth factors, the unbound growth factors were quantified by using an enzyme-linked immunosorbent assay (ELISA) according to the manufacturer's instructions, and a microplate reader (Tecan Infinite M200) was used to calculate the amount of bound growth factors.

\section{Cell seeding}

Cell experiments were performed by using mouse preosteoblast MC3T3-E1 cells purchased from Institute of Biochemistry and Cell Biology, Shanghai Institutes for Biological Sciences, Chinese Academy of Sciences. MC3T3-E1 cells were incubated in Dulbecco's modified Eagle's medium (DMEM, Gibco) supplemented with 10\% FBS (Gibco), $10 \mathrm{mM}$ HEPES (Sigma), $63 \mathrm{mg} \mathrm{L}^{-1}$ penicillin (Sigma) and $100 \mathrm{mg} \mathrm{L}^{-1}$ streptomycin (Sigma) in a humidified incubator at $37.8{ }^{\circ} \mathrm{C}$ and $5 \% \mathrm{CO}_{2}$. After 7 days in culture, the cells were harvested by trypsinization and centrifugation. Nanofibre scaffolds were sterilized in $75 \%$ ethanol for $4 \mathrm{~h}$, then washed twice with PBS, and seeded with MC3T3-E1 cells at $2.5 \times 10^{4}$ cells per $\mathrm{mL}$ per well in a 24 -well plate.

\section{Cell adhesion and proliferation}

After 1, 4 and 7 days of culture, relative cell proliferation rate were determined using MTT assay reagents according to the methods described in the literature. For the cell spreading and attachment analysis, cells were fixed in PBS containing 4\% paraformaldehyde (PFA) in PBS for 15 min followed by rinsing three times. Afterward, the MC3T3-E1 cells on the surface of the different nanofibre scaffolds were visualized was visualized by fluorescein isothiocyanate (FITC) (green) and nuclear staining (blue) using 4,6-diamidino-2-phenylindole dihydrochloride (DAPI, Invitrogen). Finally, the samples were observed by observed under a fluorescence microscope (TE2000-U, Nikon).

\section{ALP staining and activity assay}

The MC3T3-E1 cells were seeded onto the surface of PLGA, PLGA/ HA, PLGA/HA/GO, PLGA/HA/GO/bFGF, PLGA/HA/GO/BMP-2 and PLGA/HA/GO/bFGF/BMP-2 nanofibre scaffolds at a density of 2.5 $\times 10^{4}$ cells per well in 24-well plates. After incubation for 7 and 14 days on different nanofibre scaffolds, the cellular ALP activity was investigated via a pNPP assay. The absorbance at $405 \mathrm{~nm}$ was read by a multifunction microplate scanner (Infinite M200, TECAN). Measurements were normalized to the total number of cells by a BCA protein assay. For ALP staining, after 14 days culture of culture, the culture medium was removed, and MC3T3E1 cells were washed with PBS solution. Cells were then fixed in $4 \%$ paraformaldehyde in PBS for $20 \mathrm{~min}$. After washing with PBS solution, ALP incubation medium was added to each well and left to react for $30 \mathrm{~min}$ at $37{ }^{\circ} \mathrm{C}$. Thereafter, methyl green staining solution was added to stain the cells. The ALP staining results and the cell morphology were observed under a fluorescence microscope (TE2000-U, Nikon).

\section{Cell mineralization}

After induction of osteoblast differentiation induction for 21 days, the mineralization patterns of the MC3T3-E1 cells were evaluated using alizarin red S staining. Briefly, the cells were washed twice with PBS and were then fixed with $4 \%$ paraformaldehyde in PBS, pH 4.2. The fixed MC3T3-E1 cells were stained with alizarin red $\mathrm{S}$ solution $(50 \mathrm{mM})$ for $30 \mathrm{~min}$, and images were then acquired with a light microscope. Then, the ARS stained samples were washed with PBS and treated with $1 \mathrm{~mL}$ of $10 \%$ CPC (cetylpyridinium chloride) solution for calcium quantification, and the absorbance of the solution was read at $540 \mathrm{~nm}$ in a multifunction microplate scanner.

\section{Expression of osteogenesis related genes}

Total intracellular RNA was isolated from incubated cells using TRIzol reagent (Invitrogen) according to the manufacturer's protocol. The concentration and quality of the isolated RNA were evaluated using a nanodrop spectrophotometer

Table 1 List of genes and primer nucleotide sequences

\begin{tabular}{|c|c|c|}
\hline Gene annotation & Forward primer sequence & Reverse primer sequence \\
\hline OPN & TCAGGACAACAACGGAAAGGG & GGAACTTGCTTGACTATCGATCAC \\
\hline Runx2 & GCCGGGAATGATGAGAACTA & GGACCGTCCACTGTCACTTT \\
\hline GAPDH & AACTTTGGCATTGTGGAAGG & ACACATTGGGGGTAGGAACA \\
\hline
\end{tabular}


A1

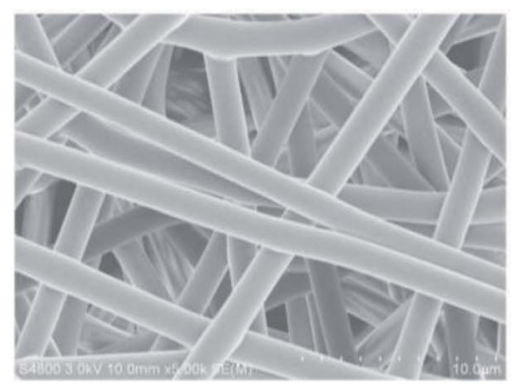

A2

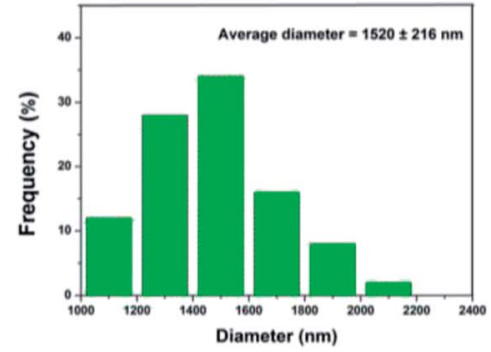

B1

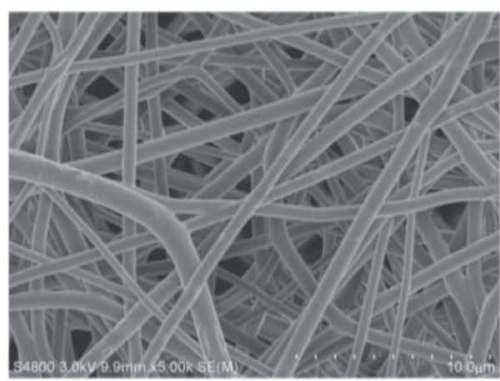

B2

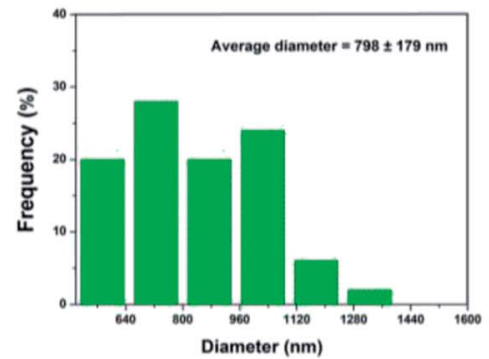

C1

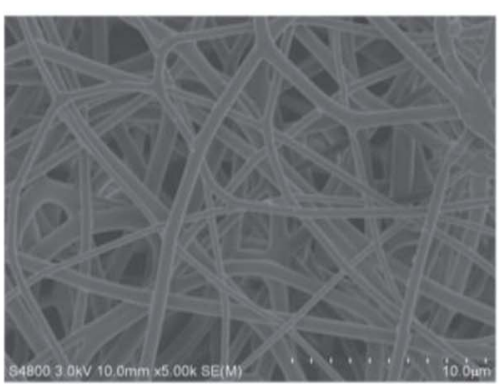

C2

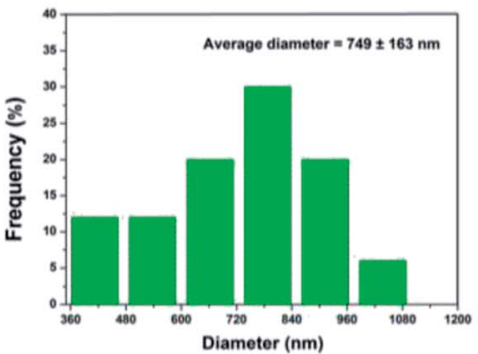

Fig. 1 SEM micrographs and nanofibre diameter distribution of (A1 and A2) PLGA, (B1 and B2) PLGA/HA and (C1 and C2) PLGA/HA/GO nanofibre scaffolds.

(Shimaduz, Japan). One milligramme of total RNA was used to produce cDNA using reverse transcription as described by the MMLV manual (Promega). The expression of osteogenic markers was quantified by a qPCR SYBRGreen Mix Kit (Takara). Target gene expression was normalized to glyceraldehyde-3phosphate dehydrogenase (GAPDH) expression. The specific primer sequences for the target gene used for qRT-PCR, including those for the anti-runt-related transcription factor 2
(RUNX2), osteopontin (OPN) and glyceraldehyde-3-phosphate dehydrogenase (GAPDH) are listed in Table 1.

\section{Statistical analysis}

All data are presented as the means \pm SDs. For analysis of multiple groups, the statistical difference was evaluated by variance analysis (one-way ANOVA, Origin 8.0). A value of $p<$ 0.05 was considered statistically significant.

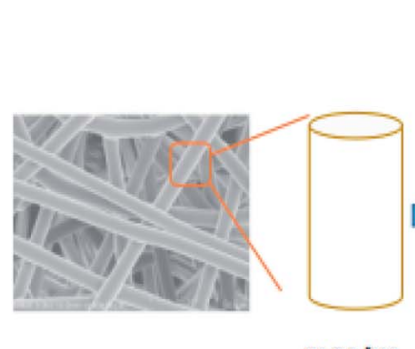

PLGA/HA

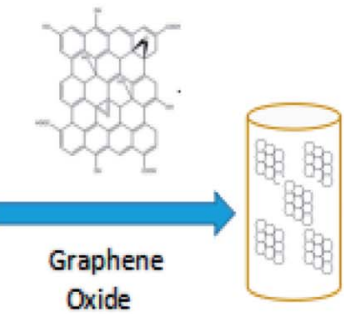

PLGA/HA/GO

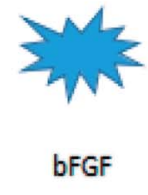

Immobilization of bFGF and BMP-2

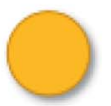

BMP-2

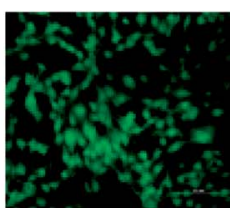

Cell proliferation

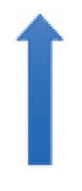

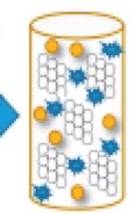

PLGA/HA/GO /bFGF/BMP-2
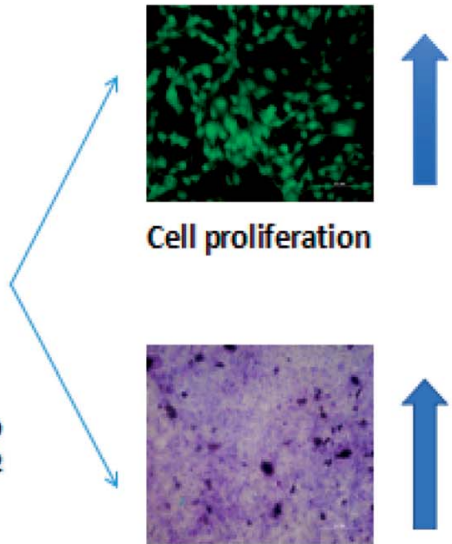

Cell differentiation

Scheme 1 Schematic illustration of BMP-2 and bFGF immobilized on PLGA/HA/GO nanofibre scaffolds for enhancing the proliferation and osteogenic differentiation of MC3T3-E1 cells. 


\section{Results and discussion}

\section{Scaffold morphology and structure}

The SEM images of different nanofibre scaffolds are shown in Fig. 1. The integration of the 3D framework composed of nanofibres is similar to the topological structure of natural ECM, and therefore would create a potential scaffold for promoting cell adhesion and growth. Uniform and smooth nanofibres with porous three-dimensional structures were fabricated from PLGA by electrospinning, and the diameter of the nanofibres was narrowly distributed, with a mean diameter $1520 \pm 216 \mathrm{~nm}$. The addition of HA and GO to PLGA resulted in a decrease in the surface roughness of the obtained composite fibres, and the average diameter dramatically decreased to 798 $\pm 179 \mathrm{~nm}$ and $749 \pm 163 \mathrm{~nm}$ when GO or HA was incorporated, likely because of the change in the conductivity and viscosity of the solution (Scheme 1).

In Fig. 1, the distribution of GO and HA on the nanofibers is barely observable, which may be due to the nanoscale size of GO and HA. Furthermore, this phenomenon can be explained partly by the fact that the GO nanosheets embedded in the nanofibers might be overlapped and aligned along the axial direction of shape anisotropy of regular shapes in nanofibers. ${ }^{32}$ To further confirm the existence of GO and HA in the nanofibre scaffolds, X-ray diffraction was used. The XRD patterns for $\mathrm{HA}$ are shown in Fig. 2, the characteristic diffraction peaks for the typical HA crystalline planes (l $\left.\begin{array}{lll}0 & 0 & 2\end{array}\right),\left(\begin{array}{l}1 \\ 1\end{array}\right.$ $\left.\begin{array}{ll}0 & 2\end{array}\right),\left(\begin{array}{lll}2 & 1 & 1\end{array}\right),\left(\begin{array}{lll}3 & 0 & 0\end{array}\right),\left(\begin{array}{lll}2 & 0 & 2\end{array}\right),\left(\begin{array}{lll}3 & 1 & 0\end{array}\right),\left(\begin{array}{lll}2 & 2 & 2\end{array}\right),\left(\begin{array}{lll}2 & 1 & 3\end{array}\right)$, and (4 $\left.\begin{array}{lll}4 & 1 & 1\end{array}\right)$ remained almost the same in the XRD patterns of the PLGA/ HA nanofibre scaffolds, demonstrating that the HA was dispersed into the PLGA only by physical mixing instead of by chemical reaction. When GO was added to the nanofibre scaffolds, the XRD patterns of the PLGA/HA/GO nanofibre scaffolds exhibited a characteristic peak at $2 \theta \approx 11^{\circ}$, corresponding to an interlayer spacing of $0.79 \mathrm{~nm}$, which is the typical separation distance of layered GO. This result indicated that the GO maintained the structure of a single twodimensional layer of carbon with oxygen-containing functional moieties within the PLGA/HA/GO nanofibre scaffolds. Taken together, the data clearly demonstrate that GO and HA were incorporated into PLGA nanofibre scaffolds.

\section{Physicochemical and mechanical characteristics of the scaffolds}

Good mechanical performance is essential for any structure used as tissue scaffold. ${ }^{34}$ The mechanical attributes of the blended nanofibre scaffolds that are made of polymers such as PLGA is not sufficient for tissue-engineering applications, but fibrous membranes using a blended polymeric system have the advantage of improved mechanical properties. Therefore, maintaining the mechanical properties of the PLGA nanofibres scaffolds is crucial for their biomedical applications, which is also anther aim of this study. Fig. 3A shows the tensile properties of the different nanofibre scaffolds, the tensile strengths of the PLGA, PLGA/HA and PLGA/HA/GO nanofibre scaffolds were approximately $6.6 \pm 0.89,7.4 \pm 2.28$ and $27.36 \pm 2.76 \mathrm{MPa}$, respectively. Compared with PLGA nanofibre scaffolds, PLGA/ HA nanofibre scaffolds were not significantly different. When GO was added to the nanofibre scaffolds, the tensile strengths of the PLGA/HA/GO nanofibre scaffolds increased in a GOdependent embedded manner, and the tensile strengths of the PLGA/HA/GO nanofibre scaffolds were 4.14 and 3.68 times higher than those of the PLGA and PLGA/HA scaffolds, respectively. This increase may be attributed to fact that the dispersion of the GO sheets in the PLGA nanofibre scaffolds produced a strong interaction between GO and the PLGA nanofibre scaffolds via $\mathrm{H}$-bonding interactions. ${ }^{35}$ Previous studies have demonstrated that the mechanical properties of the nanofibre scaffolds have influence on cell growth and differentiation. ${ }^{36,37}$ The mechanical properties of the PLGA/HA/GO nanofibre scaffolds are expected to exert a favourable influence on cell osteogenic differentiation.

Previous studies have demonstrated that the improved hydrophilicity of scaffold materials is beneficial for cell adhesion and proliferation. ${ }^{7,8}$ Therefore, one of the objectives of this study is to improve the hydrophilicity of PLGA nanofibre scaffolds in order to increase its cell adhesion and proliferation ability. As shown in Fig. 3B, the water contact angle of the control PLGA scaffold surface was greater at $(97.37 \pm 9.14)^{\circ}$ than that of the PLGA/HA scaffold, which decreased to $(85.21 \pm 2.35)^{\circ}$ due to the exposure of HA on the surface. Additionally, the incorporation of $2 \mathrm{wt} \%$ GO into PLGA/HA nanofibre scaffolds significantly decreased the water contact angle from $85.21 \pm$
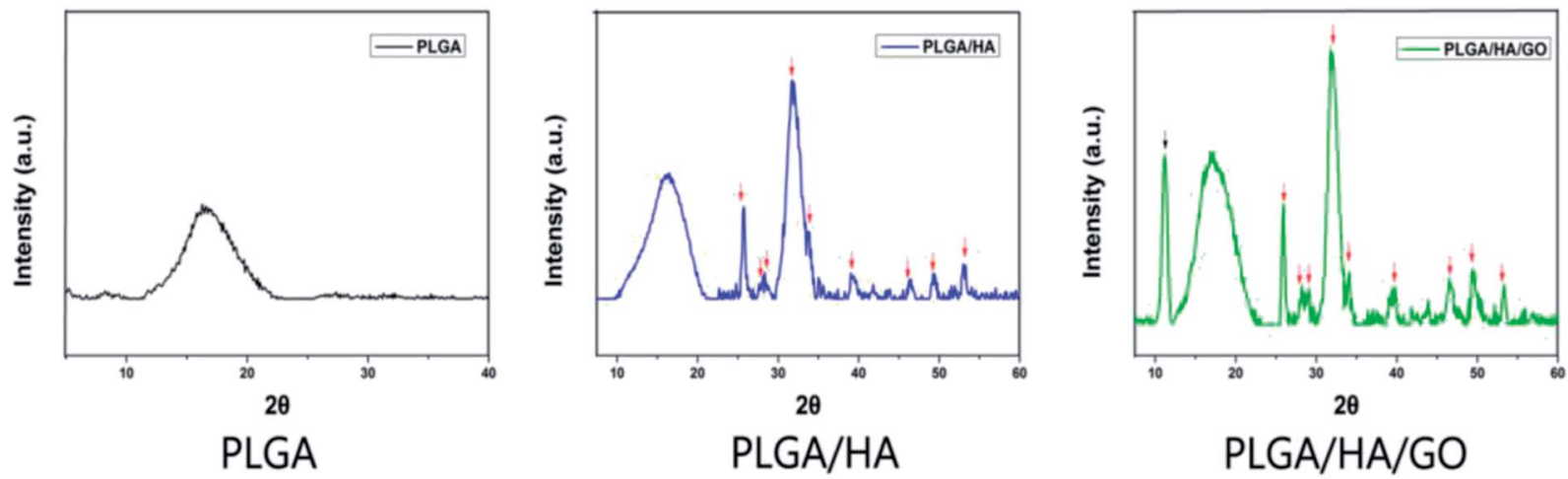

Fig. 2 XRD patterns of PLGA, PLGA/HA and PLGA/HA/GO nanofibre scaffolds. 


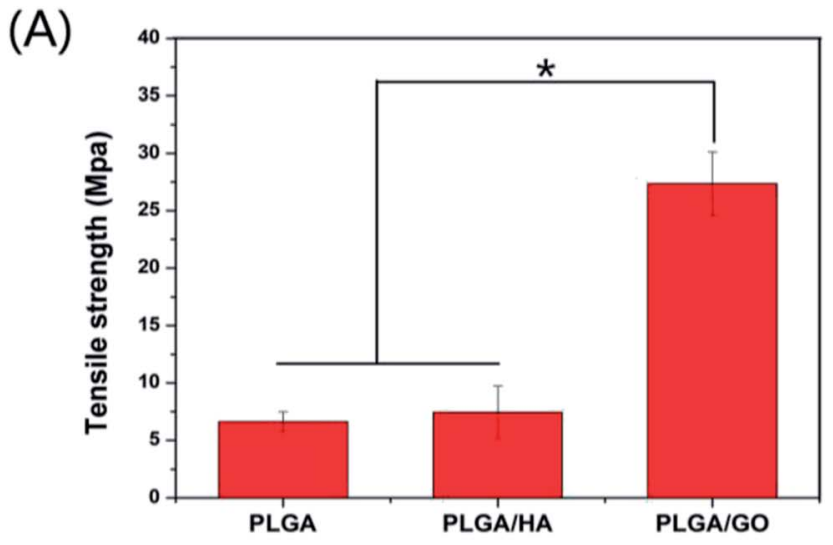

(B)

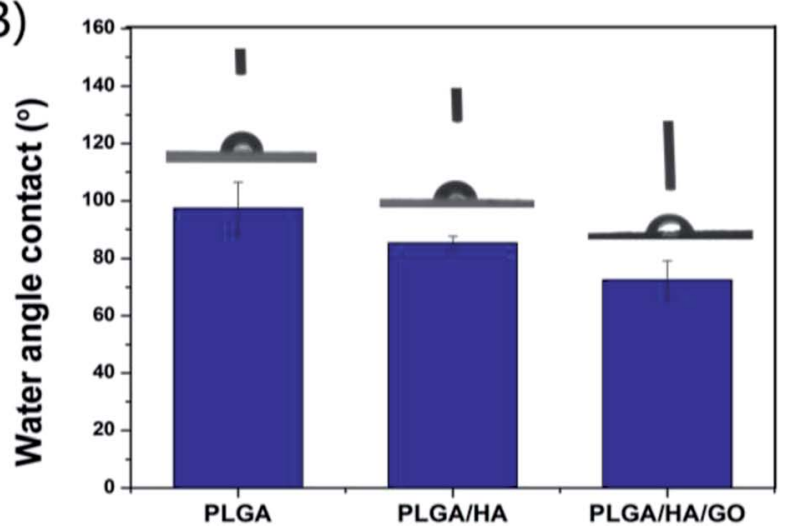

Fig. 3 (A) Tensile strength and (B) water contact angle of nanofibre scaffolds fabricated from PLGA, PLGA/HA and PLGA/HA/GO. $(n=3, p$ $<0.05$ ).

$2.35^{\circ}$ to $72.54 \pm 6.63^{\circ}$, and essentially rendered the surface hydrophilic. It is obvious that the increase in of the hydrophilicity of the PLGA/HA/GO nanofibre scaffolds should be ascribed to the presence of hydrophilic $-\mathrm{OH}, \mathrm{C}-\mathrm{O}-\mathrm{C}$ and - $\mathrm{COOH}$ groups on the GO surface. These results clearly indicated that the wettability of the nanofibre scaffold can be easily transformed by the combination of HA and GO.

\section{Bind of bFGF and BMP-2 on nanofiber scaffolds}

Growth factors can be delivered by release from a carrier. However, conventional biomaterial delivery vehicles such as PLGA/HA commonly suffer from limitations that can result in low retention of growth factors at the site of interest, supra physiological levels. As shown in Fig. 4, the bFGF and BMP-2 grafted on the different nanofibre scaffolds could be clearly detected by an enzyme-linked immunosorbent assay (ELISA). The binding efficiency of bFGF and BMP-2 to PLGA/HA/GO nanofibre scaffolds was reached to $47.32 \pm 12.87 \%$ and 59.92 $\pm 6.87 \%$, respectively; however, the binding efficiency of bFGF and BMP-2 onto PLGA/HA was only $23.97 \pm 7.71 \%$ and $31.67 \pm$ $15.21 \%$, respectively. The binding efficiency of bFGF and BMP-2 to PLGA/HA/GO nanofibre scaffolds were greater than twice the binding efficiency to the PLGA/HA nanofiber scaffold. In this study, growth factor was successfully immobilized on the nanofibre scaffold via simple dipping. GO is a nanomaterial
A

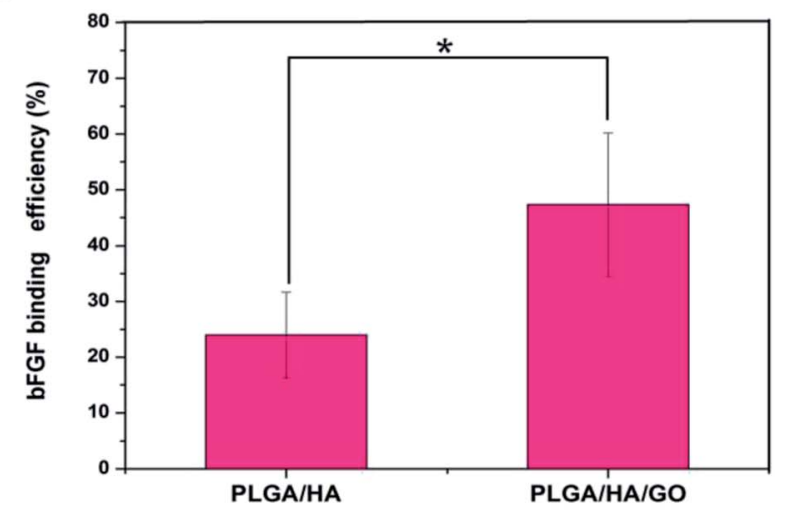

B

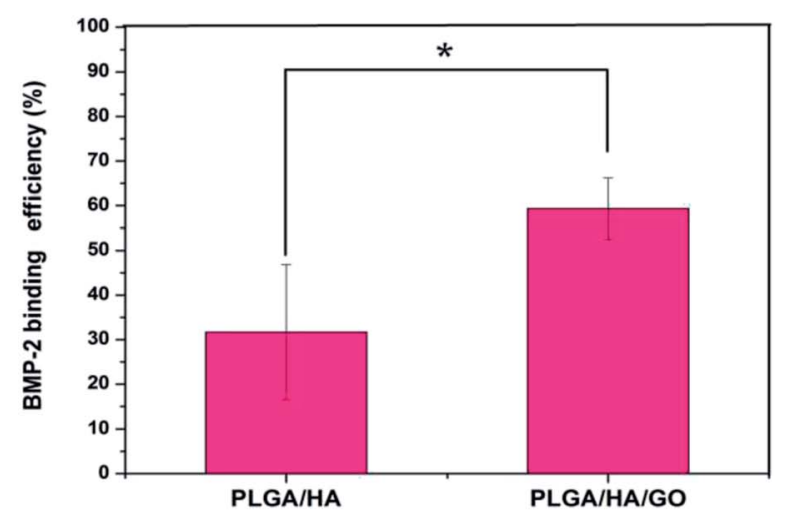

Fig. 4 Binding efficiency of bFGF (A) and BMP-2 (B) to PLGA/HA and PLGA/HA/GO nanofibre scaffolds. $p<0.05$, error bars represent the standard deviation for $n=3$.

that possesses a large surface area per unit weight. Furthermore, the surface of GO possesses a large number of - $\mathrm{COOH}$, $-\mathrm{OH}$ and $\mathrm{C}=\mathrm{O}$ groups that could enhance the adsorption of proteins by hydrogen bonding and electrostatic interactions, and thus GO can improve the polymer materials's interactions with growth factors. It was reported that GO has a strong capability to adsorb various proteins, including cytochrome $c$, bovine serum albumin, ribonuclease $\mathrm{A}$, and various growth factors. ${ }^{30,38,39}$ It was demonstrated that introducing GO onto the PLGA/HA nanofibre scaffold could increase the binding efficiency of growth factors to the nanofibre scaffold.

\section{MC3T3-E1 cell growth on nanofibre scaffolds}

Better stem cell adhesion and proliferation probably produce a larger mass of bone tissues around the scaffolds. Thus, the MC3T3-E1 cell behaviour on six kinds of nanofibre scaffolds (PLGA, PLGA/HA, PLGA/HA/GO, PLGA/HA/GO/bFGF, PLGA/HA/ GO/BMP-2 and PLGA/HA/GO/bFGF/BMP-2) was examined. The cells were seeded and cultured for 1,4 , and $7 \mathrm{~d}$ on different nanofibre scaffolds to observe the cell proliferation according to an MTT assay. As shown in Fig. 5(A), during 7 days of culture, the number of living cells increased in all groups, and showed significant growth in the PLGA/HA/GO/bFGF and PLGA/HA/GO/ bFGF/BMP-2 nanofibre scaffold groups compared with the 
other three groups. However, the PLGA/HA/GO/bFGF group showed higher cell viability than PLGA/HA/GO/BMP-2 group. The results showed that the proliferation capacity of MC3T3-E1 cells pretreated with bFGF was greater than that of MC3T3-E1 cells pretreated with BMP-2. Many studies have reported that bFGF can significantly promote cell proliferation as a therapeutic factor either alone or in combination with other active ingredients. ${ }^{\mathbf{1 4 0}}$ Furthermore, we found that the cell growth in nanofibre scaffolds containing bFGF + BMP-2 was significantly higher after 4 and 7 days than that in nanofibre scaffolds containing only bFGF or BMP-2. This observation suggests that the combination of BMP-2 and bFGF were better

$$
\text { (A) }
$$

$1 \mathrm{D}$
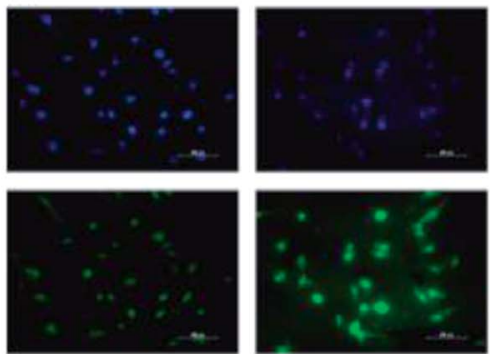

a
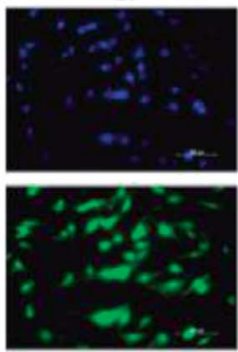

d

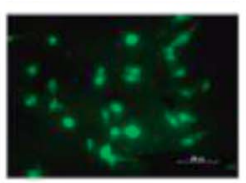

b
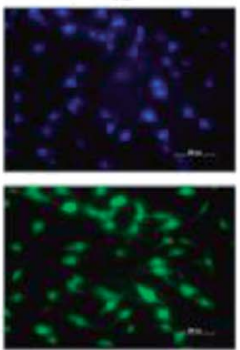

e
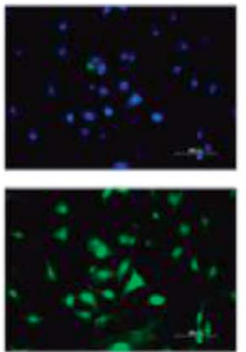

C
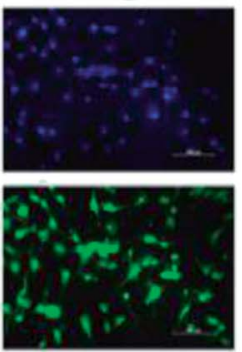

f
$3 D$
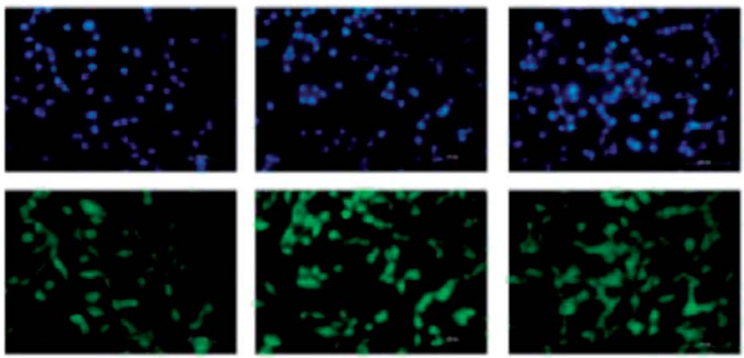

a
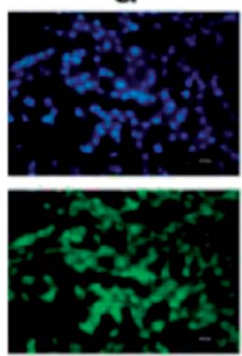

d

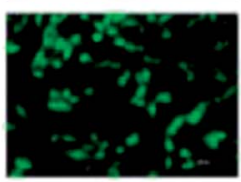

b
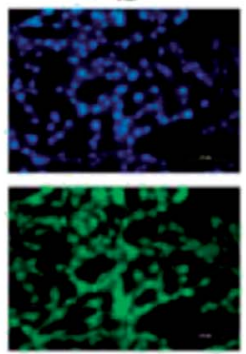

e

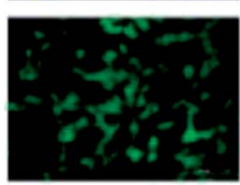

C
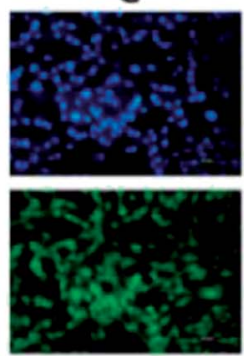

f

(B)

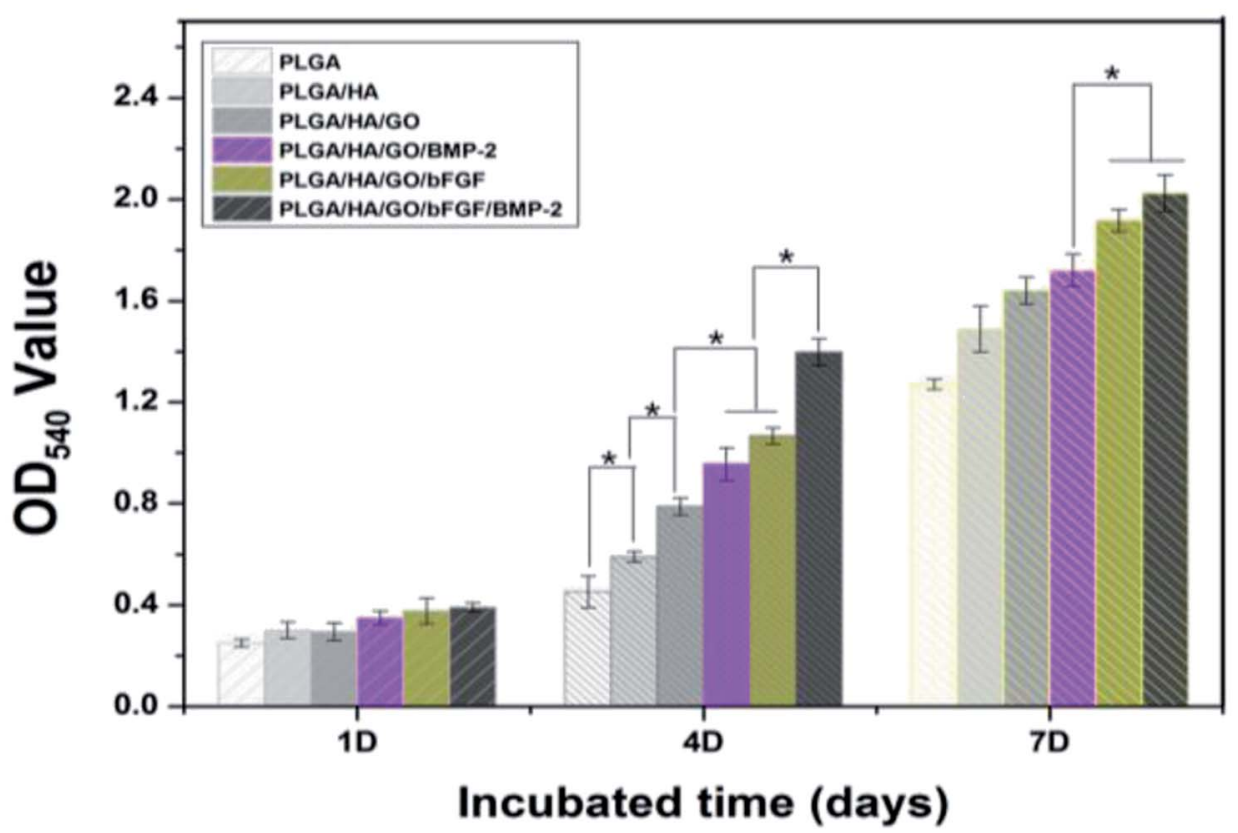

Fig. 5 (A) Fluorescence staining images of MC3T3-E1 cells cultured on different nanofibre scaffolds for 1 and 3 days: (a) PLGA, (b) PLGA/HA, (c) $\mathrm{PLGA} / \mathrm{HA} / \mathrm{GO}$, (d) PLGA/HA/GO/BMP-2, (e) PLGA/HA/GO/bFGF, and (f) PLGA/HA/GO/bFGF/BMP-2. All scale bar lengths are $100 \mu \mathrm{m}$. (B) Proliferation of MC3T3-E1 cells cultured on the nanofibre scaffolds for 1 to 7 days in vitro. ${ }^{*} p<0.05$ compared to the other groups, $n=3$. 
at promoting cell proliferation than was BMP-2 or bFGF alone. More importantly, we found that the OD values for cell viability were slightly increased in the PLGA/HA/GO nanofibre scaffolds compared with those for the PLGA and PLGA/HA nanofibre scaffolds. In the last several years, numerous studies have reported that biocompatibility and superior loading capacities of graphene materials, which are beneficial for cell proliferation and differentiation. ${ }^{28,35}$ We hypothesized that the enhanced proliferation of the MC3T3-E1 cells on the PLGA/HA/GO nanofibre scaffolds, could be due to the higher cellular protein adsorption capability of the material, the conformational change of these proteins upon the graphene-protein molecular interactions, and the higher density of oxygenated functional groups on the materials.

The cell morphology of MC3T3-E1 cells grown on the different nanofibre scaffolds was studied by cytoskeletal (green) and nuclear (blue) fluorescence staining on days 1 and 4 . As shown in Fig. 5(B), one day post-seeding, there was no significant difference in the cell number of MC3T3-E1 cells cultured on different nanofibrous matrices. However, on the surface of the PLGA/HA and PLGA/HA/GO nanofibre scaffolds, it could be seen that compared to cells on pure PLGA nanofibre scaffolds, MC3T3-E1 cells displayed an improved spreading morphology and were flattened with a larger attachment, which indicated that the incorporation of HA and GO provided a favourable cell-anchoring sites for cell adhesion. The enhancement of the cell adhesion on such nanofibre scaffolds may be attributed to the improved hydrophilicity and the $\mathrm{GO}$ functional groups such as $-\mathrm{COOH}$ and $-\mathrm{OH}$. Furthermore, the properties of HA are similar to those of the mineral phase of natural bone, which can provide a better cellular microenvironment for MC3T3-E1 cells. After bFGF and BMP-2 were immobilized, the cell adhesion on the nanofibre scaffolds was better than that on the nanofibre scaffolds without immobilized growth factors, indicating that immobilized growth factors are beneficial for cell growth and cell-cell communication. Three days post-seeding, compared to their ability to attach to the PLGA and PLGA/HA nanofibre scaffolds, MC3T3-E1 cells attached more easily to the PLGA/HA/GO nanofibre scaffolds. After bFGF and BMP-2 were immobilized, MC3T3-E1 cells were able to grow very well on the nanofibre scaffolds, and these cells were observed to be spindle-shaped and to stretch across the randomly interconnected structures. Furthermore, it was also found that the immobilization of bFGF had a greater effect than did BMP-2 on the adhesion of MC3T3-E1 cells, which was consistent with the results of the MC3T3-E1 cells proliferation assay. More importantly, the cell numbers and positive cellular interactions were the highest on the PLGA/HA/GO/bFGF/BMP-2 nanofibre scaffolds. Thus, these results indicated that both growth factors, bFGF and BMP-2, could promote the proliferation of MC3T3-E1 cells and that their combined application was better than the use of either alone.

\section{Alkaline phosphatase (ALP) activity}

Osteoblastic cells mainly proliferate for up to 7-14 days and then start to secrete ECM proteins and express early differentiation markers, such as ALP, starting after 14 days. As shown in
Fig. 6, from day 7, the differences in ALP activity between the four groups began to appear. It was found that MC3T3-E1 cells cultured on PLGA/HA/GO nanofibre scaffolds exhibited higher ALP activity than those cultured on PLGA and PLGA/HA nanofibre scaffolds, which suggested that the presence of GO stimulated an early stage of osteoblastic differentiation. Previous studies have demonstrated that GO coated titanium implants could increase ALP activity in MC3T3-E1 cells and promote MC3T3-E1 cell differentiation. Furthermore, some studies have found that the intrinsic properties of graphene can increase cytoskeletal tension, thus guiding cell behaviours such as osteogenic differentiation. ${ }^{41}$ After bFGF and BMP-2 were immobilized, the level of ALP activity in the PLGA/HA/GO/BMP2 and PLGA/HA/GO/bFGF/BMP-2 nanofibre scaffolds groups was higher than that in the other five groups $(p<0.05)$. Between the PLGA/HA/GO/bFGF/BMP-2 and PLGA/HA/GO/BMP-2 nanofibre scaffolds groups, the former had higher ALP activity, thus indicating that the combined application of BMP-2 and bFGF had a greater osteogenic induction effect than did BMP-2 alone. However, little improvement in ALP activity in MC3T3-E1 cells was found for nanofibre scaffolds treated with bFGF. The role of bFGF on osteogenic differentiation remains controversial. Several studies have reported that bFGF enhanced osteogenic differentiation in vitro. However, in this study, compared to BMP-2, we found that bFGF played a smaller role in the early osteogenic differentiation of MC3T3-E1 cells. Similarly, 14 days post-seeding, as shown in Fig. 8A, the dark blue staining in the cells cultured on PLGA/HA/GO/bFGF/BMP-2 nanofibre scaffolds was more intense in those cultured on other nanofibre scaffolds. Thus, these results indicate that the limitations of bFGF could be overcome by adding BMP-2 and the combination of bFGF and BMP-2 have synergistically enhanced the cell osteogenic differentiation.

\section{Cell mineralization}

The capacity for mineral deposition reflects osteogenesis and has been regarded as a marker for bone regeneration. We analysed the efficiency of the mineralization stage by using ARS staining as a marker for the inorganic calcium, which is a component common to bone-like structures. Fig. 7A shows the optical microscopy images of alizarin red-stained MC3T3-E1 cells on different nanofibre scaffolds on day 21. Compared to the cells grown on pure PLGA and PLGA/HA nanofibre scaffolds, the cells grown on the surface of the PLGA/HA/GO nanofibre scaffolds had an increased calcium deposition. The characteristics of graphene and its derivatives such as nanostructures, surface roughness, protein absorption ability, electrostatic interactions, and surface hydrophilicity have a great influence on the molecular pathways that control cell behavior. Previous studies have found that cells treated with GO exhibited distinctly stronger mineralization capability than did cells not treated with GO, suggesting that treatment with GO nanosheets could improve cell osteogenesis. ${ }^{33,42}$ In this study, it was also found that GO could not only promote cell proliferation but could also improve the nucleation of HA, which facilitated the late stage of cell osteogenic differentiation. After growth factors 


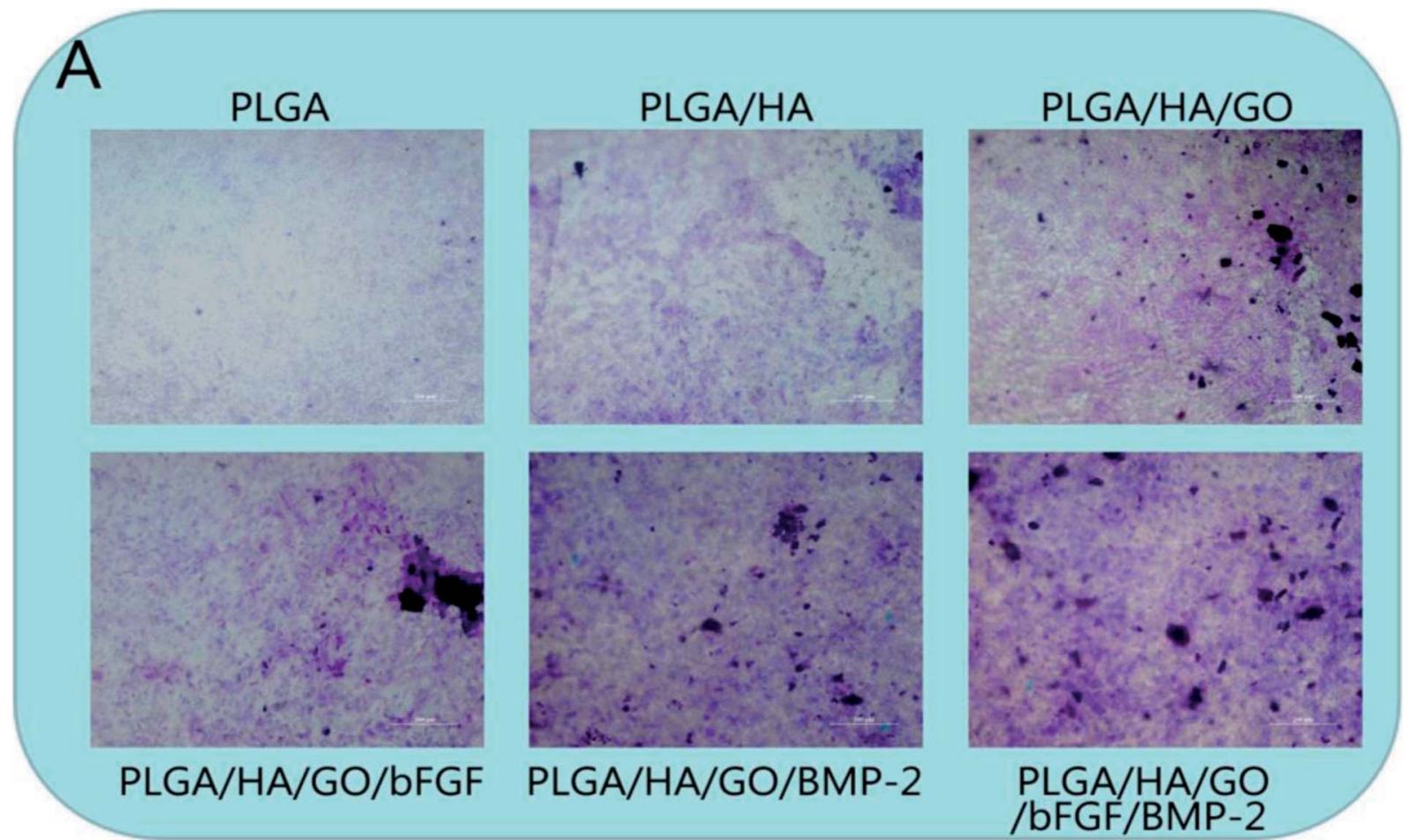

B

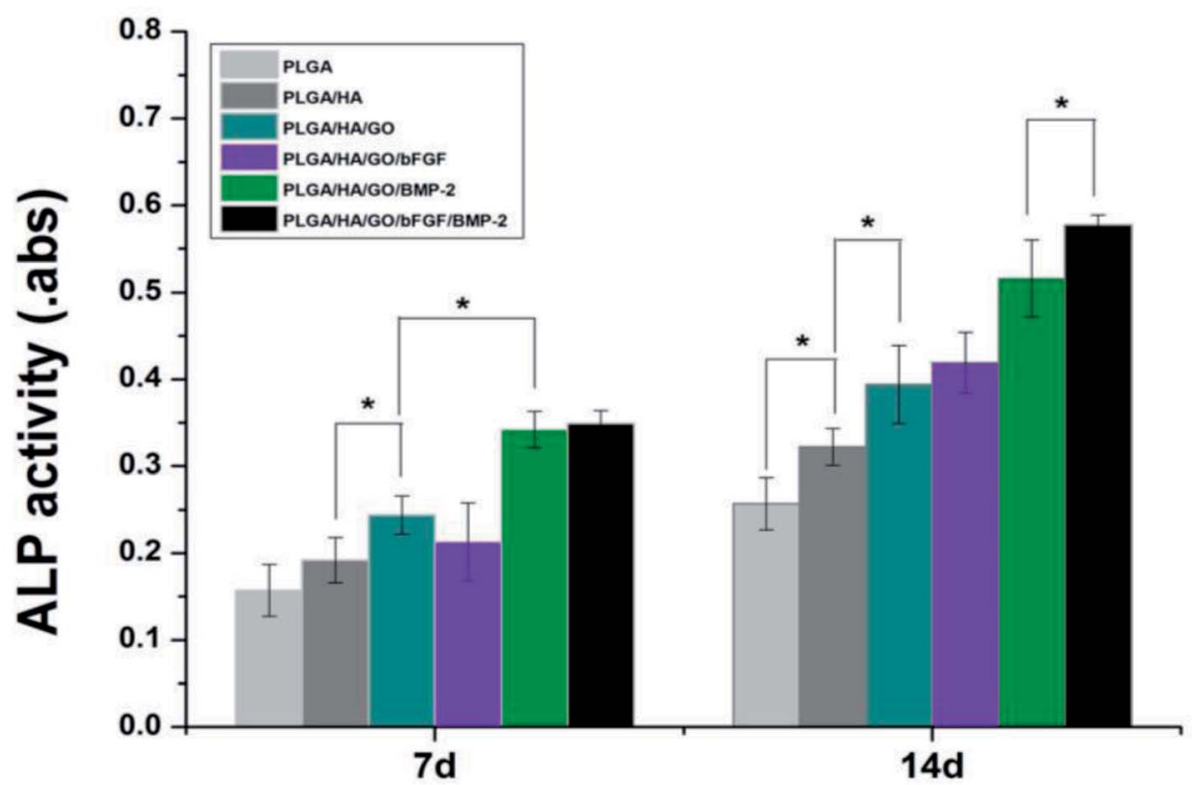

Fig. 6 (A) ALP staining images of MC3T3-E1 cells cultured on different nanofibre scaffolds for 14 days. The scale bars are all $200 \mu$ m. (B) ALP activity in MC3T3-E1 cells cultured on different nanofibre scaffolds for 7 and 14 days. $p<0.05, n=4$.

were immobilized, there was an increased calcium deposition in all growth factors-immobilized nanofibre scaffolds. However, compared with cells on nanofibre scaffolds with other immobilized growth factors, cells on bFGF immobilized nanofibre scaffolds exhibited little extracellular calcium deposition. In contrast, the analysis of cells on BMP-2 immobilized nanofibre scaffolds and on scaffolds with both immobilized BMP-2 and
bFGF demonstrated that immobilized BMP-2 possesses stronger osteoinductive ability than does bFGF. The assessment of quantitative cell mineralization was performed by extracting ARS with 10\% CPC. The total calcium content in the mineral deposits on BMP-2 immobilized nanofibre scaffolds was significantly higher than that on other nanofibre scaffolds. The above results indicated that the incorporation of bFGF and 


\section{(A)}
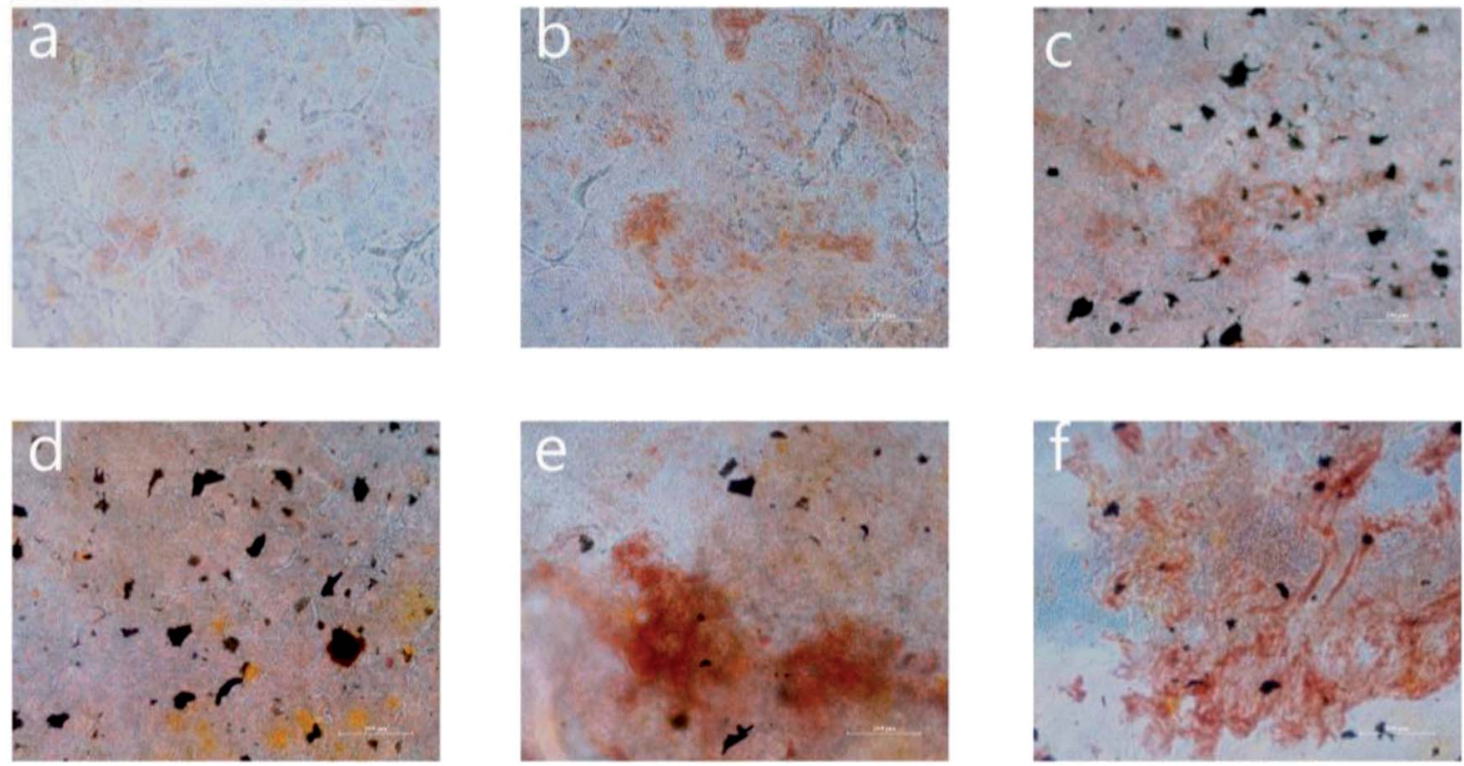

(B)

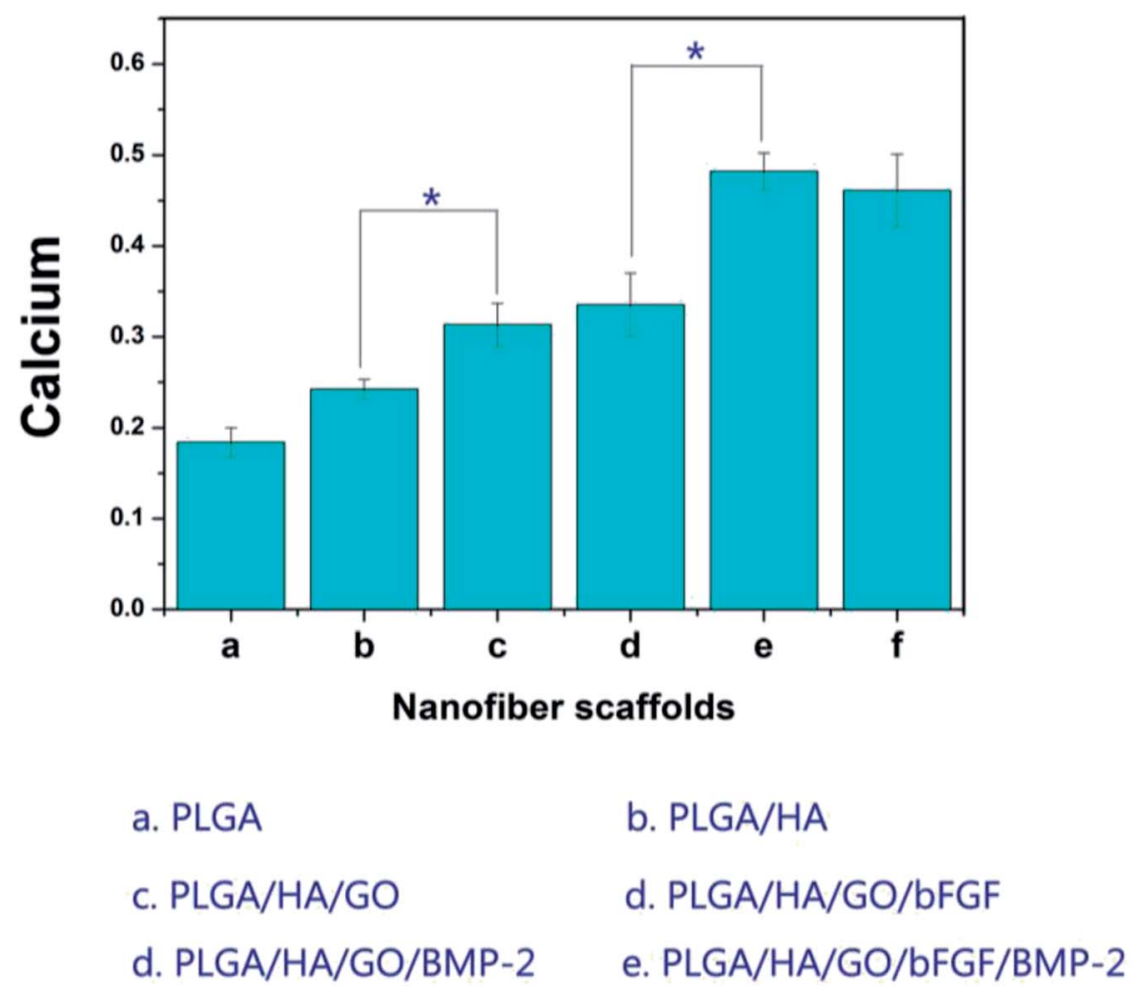

Fig. 7 (A) Alizarin red staining (a-f) and (B) the corresponding quantitative evaluation of calcium content mineral deposition in MC3T3-E1 cells cultured for three weeks. Bar $=200 \mu \mathrm{m}, * p<0.05$.

BMP-2 via GO on PLGA/HA nanofibre scaffolds significantly facilitates the calcium mineralization capacity of MC3T3-E1 cells.

\section{Quantitative real-time polymerase chain reaction}

The main characteristics of osteogenic differentiation are often accompanied by the upregulation or downregulation of certain genes such as Runx2 and OPN in each stage. ${ }^{43}$ Runx 2 is an early differentiation marker expressed during early osteogenesis, whereas OPN is usually expressed at the middle/late stages of differentiation. Therefore, the osteodifferentiation of MC3T3-E1 cells cultured on the scaffolds was also analysed by analysed the expression of several osteogenic genes via real-time PCR (Fig. 8). After $7 \mathrm{~d}$ of incubation, the expression of Runx 2 and OPN in the 
PLGA/HA/GO nanofibre scaffold group was higher than that in the PLGA and PLGA/HA/GO nanofibre scaffolds group. Such results were consistent with other observations that emphasized that the unique physicochemical characteristics of graphene oxide could plays an important role in the biological processes by influencing cell proliferation and differentiation. Recent studies demonstrated that GO can effectively regulate cell shape, adhesion, migration, proliferation and differentiation. For instance, in Liu's study, GO coated titanium implants could increase ALP activity and OCN secretion by promoting cell differentiation. ${ }^{44}$ After bFGF was immobilized, higher expression levels of Runx2 were observed in the PLGA/HA/GO/bFGF group than in the PLGA/HA/GO group. However, for the expression of OPN, there was no significant difference between the PLGA/HA/GO/bFGF and PLGA/HA/GO groups after $7 \mathrm{~d}$ of incubation. Our results demonstrate that bFGF was more effective for the enhancement of cell osteogenic differentiation at the early stage of differentiation than for the promotion of cell osteogenic differentiation at the middle/later stage of differentiation. In terms of the OPN gene, since its expression is associated with extracellular matrix mineralization and usually increases in the middle/late stages of ossification, we speculate that the bFGF was demonstrated to participate in pre-osteoblast proliferation but has a little affect during middle/later osteogenic differentiation by inducing the expression of proliferation genes. When the MC3T3-E1 cells were cultured on the PLGA/ HA/GO scaffold loaded with BMP-2 or BMP-2/bFGF, the expression levels of Runx2 and OPN were substantially higher than those in the nanofibre scaffold groups at 7 days. Furthermore, among the various nanofibre scaffolds, the PLGA/HA/GO/ bFGF/BMP-2 nanofibre scaffold was most effective at enhancing osteogenesis-related gene expression in this study, indicating that the combination of bFGF and BMP-2 had a synergistic effect in terms of cell osteogenic differentiation. From the results of the ALP activity, calcium deposition and osteogenesisrelated gene expression assays, the immobilization of BMP-2 and bFGF via GO on the PLGA/HA nanofibre scaffolds appears to be an optimal strategy for promoting osteogenic differentiation in vitro.

Bone defect healing after trauma is a critical and complex biological process after trauma. This process is regulated by various growth factors that can stimulate the production of bone growth through increasing the activation of different signalling pathways. Among the various growth factors, BMP-2 and bFGF have been proven useful for the promotion of the bone defect repair, and have been widely used in the treatment of bone defects. ${ }^{14,21}$ However, the abilities of single growth factors are limited. For example, bFGF is a proven strong cell proliferative growth factor that stimulates cell proliferation but has little effect on cell osteogenic differentiation. Thus, in this study, we postulate that a combination approach blending both BMP-2 and bFGF could enhance the differentiation/proliferative potential of the nanofibre scaffolds. The results of this study showed that the osteogenic differentiation and proliferation of MC3T3-E1 cells were enhanced by BMP-2 and bFGF, respectively. The combination of BMP-2 and bFGF retained the osteogenesis nature of BMP2 without negatively influencing the cell proliferative effect of bFGF. More importantly, it was found that the combined treatment with BMP-2 and bFGF had a better ability to promote bone regeneration than did the single use of either BMP-2 or bFGF alone, thus indicating that the combined use of BMP-2 and bFGF had a better effect on promoting bone regeneration. Furthermore, in this study, we designed a method for grafting BMP-2 and bFGF onto the surface of PLGA/HA nanofiber scaffolds by the infusion of GO. This novel method seems to be relatively simple, stable, effective and mild for the modification conditions

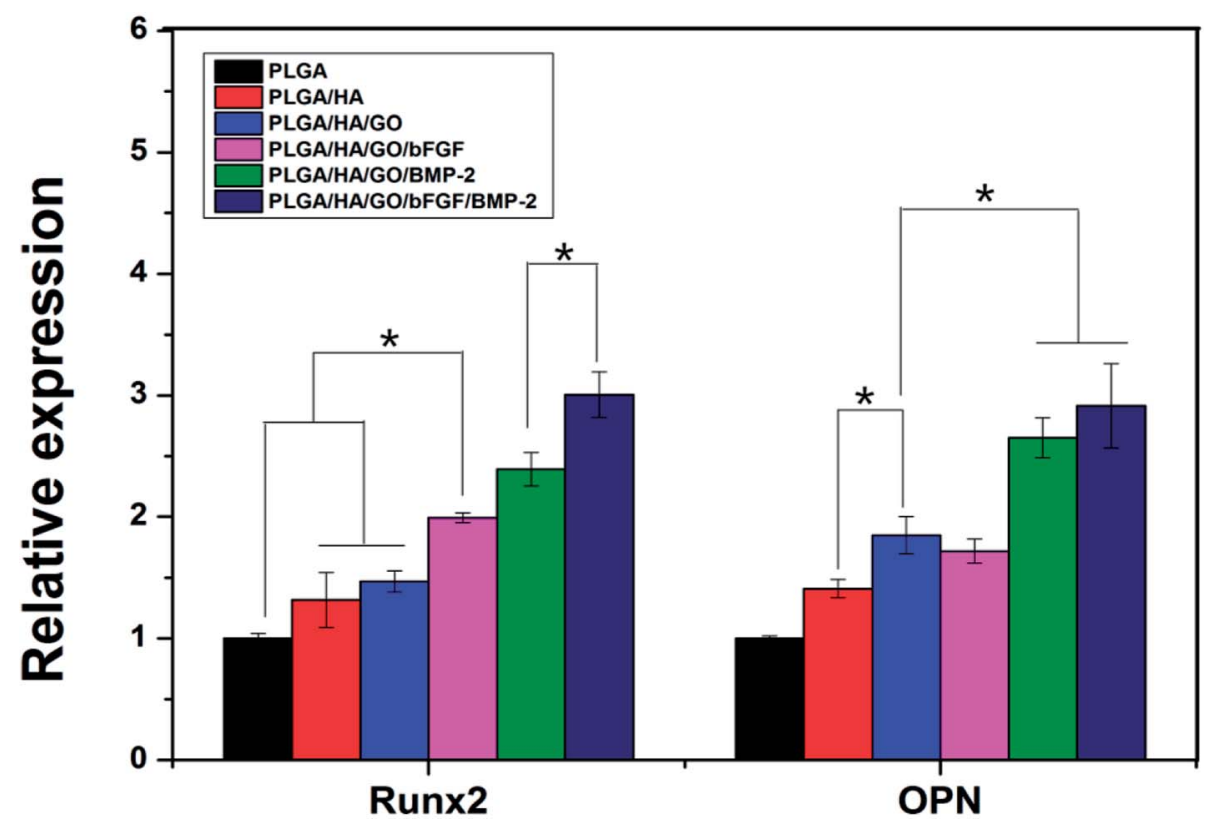

Fig. 8 The qRT-PCR analysis for Runx2 and OPN expression in MC3T3-E1 cells cultured on the different nanofibre scaffolds for $7 \mathrm{~d}$. * Indicates significant differences $(p<0.05)$. 
compared to the traditional methods for the combination use of growth factors and polymer scaffold such as direct blending and simple physical adsorption. It was found that introducing GO onto a PLGA scaffold could effectively increase the binding efficiency of growth factors to the scaffold. Furthermore, the infusion of GO could also effectively enhance the surface properties of PLGA/HA nanofibre scaffolds, such as the hydrophilicity and mechanical properties, which can provides a better cellular microenvironment for cell adhesion, proliferation and osteogenic differentiation. Therefore, we speculate that the impregnation of graphene oxide into PLGA/HA/GO nanofibre scaffolds is an efficient system for the synergistic delivery of multiple growth factors. In the future, medical biodegradable polymer scaffolds with the desirable surface biological activities achieved in our study will hold great practical potential for further scale-up in clinical application, in particular as a kind of orthopedic implants. However, an in vivo study of the biodegradable nanofibre scaffolds is necessary in order to achieve more conclusive outcomes for bone defect therapy by overcome the current drawbacks and promoting the clinical applications of this novel technique. We believe that this work can provide a better understanding of the biological applications of engineered graphene-based nanomaterials.

\section{Conclusions}

In this study, PLGA/HA/GO nanofibre scaffolds were constructed to deliver BMP-2 and bFGF in combination for bone defect repair. The incorporation of $2 \mathrm{wt} \%$ GO into the PLGA/HA nanofibre scaffolds dramatically accelerated the binding affinity of the PLGA/HA nanofibre scaffolds for bFGF and BMP-2, which can effectively enhance bFGF and BMP-2 binding on the surface of the PLGA/HA nanofibre scaffold. The mechanical properties and hydrophilicity of PLGA/HA nanofibre scaffolds were also significantly enhanced through the introduction of GO. In addition, from the results of the ALP activity, mineral deposition and osteogenic gene expression assays, the results indicated that PLGA/HA/GO/bFGF/BMP-2 composite scaffolds not only support MC3T3-E1 cells adhesion and proliferation but also enhance osteogenesis and alkaline phosphatase activity. A facile combination of BMP-2 and bFGF in the PLGA/HA/GO nanofibre scaffolds could therefore result in a promising nanofibre scaffold for bone tissue development, with BMP-2 inducing differentiation and bFGF promoting proliferation. Based on the excellent mechanical properties, osteoinductivity and regenerative activity, these nanofibre scaffolds are a promising substrate for the repair of bone defects.

\section{Conflicts of interest}

The authors declare no competing financial interest.

\section{Acknowledgements}

This study was supported by a grant from National Natural Science Foundation of China (No. 81672263, No. 31572217).

\section{References}

1 W. L. Shao, J. X. He, F. Sang, Q. Wang, L. Chen, S. Z. Cui and B. Ding, Mater. Sci. Eng., C, 2016, 62, 823-834.

2 S. E. Kim, Y. P. Yun, Y. K. Han, D. W. Lee, J. Y. Ohe, B. S. Lee, H. R. Song, K. Park and B. J. Choi, Carbohydr. Polym., 2014, 99, 700-709.

3 T. Gao, N. Zhang, Z. Wang, Y. Wang, Y. Liu, Y. Ito and P. Zhang, Macromol. Biosci., 2015, 15, 1070-1080.

4 O. Kozawa, M. Niwa, H. Matsuno, A. Ishisaki, K. Kato and T. Uematsu, Arch. Biochem. Biophys., 2001, 388, 237-242.

5 X. Qu, F. He, H. Q. Tan, Y. M. Yu, A. Axrap, M. Wang, K. Dai, Z. Zhang, F. Yang, S. G. Wang, J. Kohn and C. S. Liu, J. Mater. Chem. B, 2016, 4, 4901-4912.

6 G. Bhakta, B. Rai, Z. X. H. Lim, J. H. Hui, G. S. Stein, A. J. van Wijnen, V. Nurcombe, G. D. Prestwich and S. M. Cool, Biomaterials, 2012, 33, 6113-6122.

7 Z. L. Wang, L. Chen, Y. Wang, X. S. Chen and P. B. Zhang, ACS Appl. Mater. Interfaces, 2016, 8, 26559-26569.

8 T. L. Gao, W. W. Cui, Z. L. Wang, Y. Wang, Y. Liu, P. S. Malliappan, Y. Ito and P. B. Zhang, RSC Adv., 2016, 6, 20202-20210.

9 Y. Takahashi, M. Yamamoto and Y. Tabata, Biomaterials, 2005, 26, 4856-4865.

10 K. T. Peng, M. Y. Hsieh, C. T. Lin, C. F. Chen, M. S. Lee, Y. Y. Huang and P. J. Chang, BMC Musculoskeletal Disord., 2016, 17, 286.

11 S. Park, H. A. Heo, K. B. Lee, H. G. Kim and S. W. Pyo, Implant Dentistry, 2017, 26, 4-11.

12 X. F. Shen, Y. X. Zhang, Y. Gu, Y. Xu, Y. Liu, B. Li and L. Chen, Biomaterials, 2016, 106, 205-216.

13 M. L. Radomsky, T. B. Aufdemorte, L. D. Swain, W. C. Fox, R. C. Spiro and J. W. Poser, J. Orthop. Res., 1999, 17, 607-614.

14 M. Du, T. Zhu, X. Q. Duan, S. H. Ge, N. Li, Q. F. Sun and P. S. Yang, Mater. Sci. Eng., C, 2017, 70, 62-70.

15 J. S. Su, H. Z. Xu, J. Sun, X. Gong and H. Zhao, Int. J. Mol. Sci., 2013, 14, 12714-12728.

16 L. Feng, Y. H. Li, W. C. Zeng, B. Xia, D. S. Zhou and J. Zhou, Exp. Ther. Med., 2017, 14, 6087-6092.

17 W. Qi, J. Yan, H. F. Sun and H. Wang, ACS Omega, 2017, 2, 899-909.

18 Y. N. Yan, Z. Xiong, Y. Y. Hu, S. G. Wang, R. J. Zhang and C. Zhang, Mater. Lett., 2003, 57, 2623-2628.

19 S. G. Wang, Q. Cai, J. W. Hou, J. Z. Bei, T. Zhang, J. Yang and Y. Q. Wan, J. Biomed. Mater. Res., 2003, 66A, 522-531.

20 Z. Xiong, Y. N. Yan, S. G. Wang, R. J. Zhang and C. Zhang, Scr. Mater., 2002, 46, 771-776.

21 C. M. Cowan, T. Aghaloo, Y. F. Chou, B. Walder, X. L. Zhang, C. Soo, K. Ting and B. Wu, Tissue Eng., 2007, 13, 501-512.

22 H. Shen, X. X. Hu, J. Z. Bei and S. G. Wang, Biomaterials, 2008, 29, 2388-2399.

23 J. Wu, L. Li, C. Fu, F. Yang, Z. Jiao, X. Shi, Y. Ito, Z. Wang, Q. Liu and P. Zhang, Colloids Surf., B, 2018, 169, 233-241.

24 R. Wang, D. Shou, O. Lv, Y. Kong, L. H. Deng and J. Shen, Int. J. Biol. Macromol., 2017, 103, 248-253. 
25 J. Q. Chen, C. B. Liu, G. Zeng, Y. J. You, H. N. Wang, M. Yan, R. Q. Zheng and L. Song, Nanomedicine, 2016, 12, 515.

26 X. X. Yao, Z. F. Tian, J. X. Liu, Y. F. Zhu and N. Hanagata, Langmuir, 2017, 33, 591-599.

27 S. Mohammadi, S. S. Shafiei, M. Asadi-Eydivand, M. Ardeshir and M. Solati-Hashjin, J. Bioact. Compat. Polym., 2017, 32, 325-342.

28 C. Y. Liang, Y. C. Luo, G. D. Yang, D. Xia, L. Liu, X. M. Zhang and H. S. Wang, Nanoscale Res. Lett., 2018, 13, 15.

29 Y. C. Shin, L. Jin, J. H. Lee, S. Jun, S. W. Hong, C. S. Kim, Y. J. Kim, J. K. Hyun and D. W. Han, Sci. Adv. Mater., 2017, 9, 232-237.

30 W. G. La, M. J. Jung, J. K. Yoon, S. H. Bhang, H. K. Jang, T. J. Lee, H. H. Yoon, J. Y. Shin and B. S. Kim, Carbon, 2014, 78, 428-438.

31 C. H. Zhao, S. Pandit, Y. F. Fu, I. Mijakovic, A. Jesorka and J. H. Liu, RSC Adv., 2016, 6, 38124-38134.

32 Y. Luo, H. Shen, Y. X. Fang, Y. H. Cao, J. Huang, M. X. Zhang, J. W. Dai, X. Y. Shi and Z. J. Zhang, ACS Appl. Mater. Interfaces, 2015, 7, 6331-6339.

33 H. Y. Liu, J. Cheng, F. J. Chen, D. C. Bai, C. W. Shao, J. Wang, P. X. Xi and Z. Z. Zeng, Nanoscale, 2014, 6, 5315-5322.

34 Z. X. Meng, Q. T. Zeng, Z. Z. Sun, X. X. Xu, Y. S. Wang, W. Zheng and Y. F. Zheng, Colloids Surf., B, 2012, 94, 44-50.
35 O. J. Yoon, C. Y. Jung, I. Y. Sohn, H. J. Kim, B. Hong, M. S. Jhon and N. E. Lee, Composites, Part A, 2011, 42, 1978-1984.

36 J. Wala, D. Maji and S. Das, Biomed. Mater., 2017, 12, 065002. 37 S. K. Seidlits, Z. Z. Khaing, R. R. Petersen, J. D. Nickels, J. E. Vanscoy, J. B. Shear and C. E. Schmidt, Biomaterials, 2010, 31, 3930-3940.

38 M. Patila, L. V. Pavlidis, A. Kouloumpis, K. Dimos, K. Spyrou, P. Katapodis, D. Gournis and H. Stamatis, Int. J. Biol. Macromol., 2016, 84, 227-235.

39 B. Cai, K. B. Hu, C. M. Li, J. Jin and Y. X. Hu, Appl. Surf. Sci., 2015, 356, 844-851.

40 X. M. Sun, L. Y. Cheng, J. W. Zhao, R. Jin, B. S. Sun, Y. M. Shi, L. Zhang, Y. G. Zhang and W. G. Cui, J. Mater. Chem. B, 2014, 2, 3636-3645.

41 S. W. Crowder, D. Prasai, R. Rath, D. A. Balikov, H. Bae, K. I. Bolotin and H. J. Sung, Nanoscale, 2013, 5, 4171-4176.

42 R. Isseroff, J. Chen, Z. Khan, A. Guha, S. Lin, J. Y. Li, K. C. Fang, L. X. Zhang, M. Simon and M. Rafailovich, MRS Adv., 2018, 3, 1725-1733.

43 B. Setzer, M. Bachle, M. C. Metzger and R. J. Kohal, Biomaterials, 2009, 30, 979-990.

44 M. T. Liu, L. Y. Hao, Q. Huang, D. Zhao, Q. S. Li and X. X. Cai, J. Nanosci. Nanotechnol., 2018, 18, 3134-3140. 